INPLASY

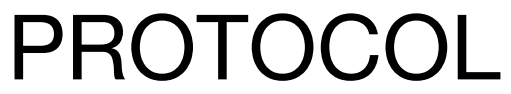

To cite: Enríquez-Enríquez et al. Warm-up strategies and performance in competitive swimmers. A systematized narrative review. Inplasy protocol 202190038. doi: 10.37766/inplasy2021.9.0038

Received: 13 September 2021

Published: 13 September 2021

Corresponding author:

Francisco Guede-Rojas

francisco.guede@unab.cl

Author Affiliation:

Universidad Andres Bello.

Support: None.

Review Stage at time of this submission: Risk of bias assessment.

Conflicts of interest: None declared.

\section{Warm-up strategies and performance in competitive swimmers. A systematized narrative review}

Enríquez-Enríquez, D1; Mecina-Zapata C2; Riveros-Cárcamo, H3; Jerez-Mayorga, D4; Guede-Rojas, F5.

Review question / Objective: In competitive swimmers, are warm-up strategies effective in improving time trial performance? The aim of this systematized narrative review is to analyze the state of the art regarding the effectiveness of warm-up strategies on time trial performance in competitive swimmers.

Condition being studied: Effects of active, passive or mixed warm-up strategies on performance in time trials equal to or less than $\mathbf{2 0 0}$ meters applied in healthy competitive swimmers over 15 years of age.

Information sources: An electronic search of the MEDLINE database was performed through PubMed.

INPLASY registration number: This protocol was registered with the International Platform of Registered Systematic Review and Meta-Analysis Protocols (INPLASY) on 13 September 2021 and was last updated on 13 September 2021 (registration number INPLASY202190038).

\section{INTRODUCTION}

Review question / Objective: In competitive swimmers, are warm-up strategies effective in improving time trial performance? The aim of this systematized narrative review is to analyze the state of the art regarding the effectiveness of warm-up strategies on time trial performance in competitive swimmers.

Rationale: Although warm-up is a common practice prior to competitive swimming, there is little evidence of its efficacy and it has mainly focused on warm-up in water. In this regard, a narrative review published in 
2014 described the effects of warm-up on performance in swimmers and proposed optimized warm-up strategies. However, research addressing dryland exercises or passive modalities was scarce and inconclusive. In addition, the effects of the combination of warm-up strategies on performance were not addressed. Therefore, the aim of this systematized narrative review is to analyze the state of the art regarding the effectiveness of warm-up strategies on time trial performance in competitive swimmers.

Condition being studied: Effects of active, passive or mixed warm-up strategies on performance in time trials equal to or less than 200 meters applied in healthy competitive swimmers over 15 years of age.

\section{METHODS}

Search strategy: Population (\#1): "elite swimming" OR swimming OR "international swimmers" OR swim OR athletes[MeSH Terms] OR swimming[MeSH Terms]. Intervention (\#2): "warm-up strategies" OR "additional warm-up activities" OR "transition phase" OR warm-up OR warmup exercise[MeSH Terms]. Outcomes (\#3): "sports performance" OR performance OR athletic performance[MeSH Terms]. Last search operation: \#1 AND \#2 AND \#3 in "All fields".

Participant or population: Healthy swimmers over 15 years of age, who participate in regional, national or international competitions.

Intervention: Active, passive or mixed warm-up strategies, carried out in or out of the water, followed by a transition phase.

Comparator: Unrestricted control
conditions.

Study designs to be included: Experimental designs.

Eligibility criteria: (a). Inclusion criteria: Population: healthy swimmers over 15 years of age, participating in regional, national or international competitions. Intervention: Active, passive or mixed warm-up strategies, performed in or out of the water, followed by transition phase. Comparison: Unrestricted control conditions. Outcomes: Performance in a time trial less than or equal to $200 \mathrm{~m}$. Time: Studies published between 2013 and 2020. Types of studies: Experimental designs. (b). Exclusion criteria: We excluded studies that: (I) were conducted in open water; (II) considered submaximal testing or testing to exhaustion; (III) focused on recovery between two or more time trials. In addition, the following were excluded: protocols, secondary studies, and conference reports.

Information sources: An electronic search of the MEDLINE database was performed through PubMed.

\section{Main outcome(s): Time trial performance.}

Additional outcome(s): None.

Data management: Three independent reviewers carried out a selection process that initially considered a screening by titles and abstracts, and subsequently the exhaustive review of the full text (eligibility). Finally, the reviewers extracted relevant data from the included studies, using a form that considered: characteristics of the methodological design, authors' names, population, intervention and results. Possible conflicts at each stage of study selection and data extraction were resolved by consensus of all authors.

Quality assessment / Risk of bias analysis: The methodological quality of the included articles will be evaluated using the 10-point PEDro scale, using the following criteria: 1-3 points for low quality, 4-6 points for moderate quality, above 7 points for high quality.

Strategy of data synthesis: None.

Subgroup analysis: None.

Sensitivity analysis: None. 
Language: English or Spanish.

Country(ies) involved: Chile.

Other relevant information: None.

Keywords: swimming; warm-up exercise; athletic performance.

Dissemination plans: Peer-reviewed indexed journal.

Contributions of each author:

Author 1 - Diego Enríquez-Enríquez Literature search and selection. Quality rating. Draft the manuscript.

Email: d.enriquez.e@gmail.com

Author 2 - Carlos Mecina-Zapata Literature search and selection. Quality rating.

Email: cmecina.z@gmail.com

Author 3 - Hernán Riveros-Cárcamo Literature search and selection.

Email: h.riveroscarcamo@gmail.com

Author 4 - Daniel Jerez-Mayorga Reviewer the manuscript.

Email: daniel.jerez@unab.cl

Author 5 - Francisco Guede-Rojas - Study design and guidance.

Email: francisco.guede@unab.cl 